\title{
Recovery of total I-131 activity within focal volumes using SPECT and 3D OSEM
}

\author{
Kenneth F Koral, Anastasia Yendiki and Yuni K Dewaraja \\ School of Medicine, University of Michigan, Ann Arbor, MI 48109-0552, USA
}

Received 5 September 2006, in final form 15 November 2006

Published 15 January 2007

Online at stacks.iop.org/PMB/52/777

\begin{abstract}
We experimentally investigated the SPECT recovery of I-131 activity in multiple spheres located simultaneously at different locations within a cylindrical phantom that had an elliptical cross section. The sphere volumes ranged from $209 \mathrm{cc}$ down to $4.2 \mathrm{cc}$. A Prism 3000 camera and two types of parallel-hexagonal-hole collimation were employed: high energy (HE) and ultra high energy (UHE). Using appropriately-different 3D models of the point source response function for the two types of collimation, approximately the same recovery of activity could be achieved with either collimation by 3D OSEM reconstruction. The recovery coefficient was greater with no background activity in the phantom by 0.10 , on average, compared to that with background. In the HE collimation case, the activity recovery was considerably better for all volumes using 3D OSEM reconstruction than it had been in the past using 1D SAGE reconstruction. Recovery-coefficient-based correction in a simulated patient case involving spherical tumours moderately improved the activity estimates (average error reduced from 14\% to $9 \%$ for UHE collimation, and from $15 \%$ to $11 \%$ for HE collimation). For a test case with HE collimation, increasing the projection-image sampling density while decreasing the image voxel size increased the recovery coefficient by 0.075 on average, and, if used in a full set of calibration measurements of recovery coefficient versus volume, might lead to further improvement in accuracy for the patient case.
\end{abstract}

\section{Introduction}

To accurately estimate the total radiation absorbed dose to a tumour during therapy with an I-131 radiopharmaceutical, one must primarily quantify the total activity within the tumour volume accurately, because most of the energy deposition within the tumour comes from local beta particles. Accurately quantifying the activity distribution within the tumour, and/or for the entire field-of-view affects the tumour gamma dose and so is helpful, but secondary, for an accurate estimate of the spatially-averaged radiation absorbed dose. Reconstruction using a basic, unregularized ordered subsets expectation maximization (OSEM) algorithm (Hudson 
and Larkin 1994) without post-reconstruction filtering is indicated for accurately estimating total activity in focal targets surrounded by a low level of background activity because, without regularization and without the post-filtering considered by others (Beekman et al 1998, Vija and Engdahl 2003), counts should be correctly located, that is, not move from target into background and vice versa due to 'smoothing'.

In addition, there is evidence that three-dimensional (3D) modelling (Zeng and Gullberg 1992) of the collimator-detector response yields resolution improvement:

(a) In an analytical simulation of a cylindrical annulus imaged with fan-beam collimation, 50 iterations of the expectation maximization (EM) reconstruction algorithm with 3D modelling of the point-source-response function (psrf) yielded a sharper image than without the modelling (Zeng et al 1991).

(b) Furthermore, for a hot-rod circular Data Spectrum Inc. phantom filled with 99m-Tc, adding 3D modelling to an EM reconstruction that included attenuation correction clearly yielded, for example, a better-resolved inner rod in the third sector compared to results without the modelling (Zeng et al 1991).

(c) In I-131 research, unregularized OSEM with a 3D model produced 1.3 times more counts in a $200 \mathrm{cc}$ sphere compared to a regularized space-alternating general expectation maximizing (SAGE) algorithm with a 1D model (Fessler and Hero 1994). In this case there was zero background. No post-reconstruction filtering was used in either case. The relative-counts factor decreased as the background increased, mostly because the counts located in the sphere by $1 \mathrm{D}$ SAGE reconstruction were increasing with background (Koral et al 2004). The increase was presumably due to count spill in.

(d) Monte Carlo simulation of I-131 data yielded very good activity recovery for spheres smaller than $200 \mathrm{cc}$ (Koral et al 2004). However, the simulation covered only successive cases of an isolated sphere of a given size at the very centre of the elliptical cross section of the cylindrical phantom.

In this paper, we experimentally measure the I-131 psrf at different depths to construct a 3D model of the collimator-detector response. We then acquire data for a cylindrical phantom containing spheres of different sizes. We reconstruct that data using a larger number of iterations of 3D OSEM than is usually employed by others. Also, as mentioned previously, we do not employ conventional, post-reconstruction Gaussian filtering. The resultant images are noisy as a result. However, we can easily interpret them because we have a superimposed CT to use to set the volumes of interest (VoIs) for the spheres. The total count in each VoI asymptotically approaches a converged value after 100 iterations. The recovery coefficient, $\mathrm{RC}$, for each sphere is defined as its estimated activity over its true activity. The estimated activity for each sphere is determined from the converged value for its total count and a countsto-activity conversion factor, $\mathrm{CF}$, that is consistent for all the spheres in the given phantom test. This $\mathrm{CF}$ is calculated from the converged value for the total count in the largest sphere and its true activity.

An application for measuring $\mathrm{RC}$ versus sphere volume, $V$, is to use the determined dependence to correct apparent activities in focal volumes measured subsequently. The procedure is to divide the apparent activity by the RC value appropriate for the target volume to obtain the corrected activity (Koral et al 2003). In the simplest approach, the dependence from a uniformly-attenuating phantom of a given size and shape is applied to a non-uniformly attenuating patient who can have a different size and shape. In this paper, we use this approach to show, with previously-published Monte Carlo patient simulations (Dewaraja et al 2005), that correction based on the RC values determined herein can be beneficial. 


\section{Materials and methods}

\section{Measurement using $64 \times 64$ projection sampling and $7.6 \mathrm{~mm}$ image voxel}

I-131 has a principal photon emission at $364 \mathrm{keV}(90.5 \%)$ and smaller-strength emissions at $637 \mathrm{keV}(7.1 \%)$ and $723 \mathrm{keV}(1.9 \%)$. Data to establish the 3D model for OSEM reconstruction were obtained by sequentially placing a point source at five distances in front of one head of a Marconi Medical Prism 3000 triple-headed scintillation camera. The image obtained at a given distance is called the psrf. The camera was collimated by a high-energy (HE) and by an ultra-high-energy (UHE) parallel-hexagonal-hole collimator for separate psrf measurements. (The UHE collimator was designed for positron imaging and thus had thicker septa that greatly reduced septal penetration from I-131.) The five distances ranged from $2.2 \mathrm{~cm}$ to $24.6 \mathrm{~cm}$. The planar acquisitions were carried out into a $512 \times 512$ matrix.

For the psrf acquisitions, and also for the SPECT acquisitions, three energy windows were employed: a $20 \%$ window visually centred on the $364 \mathrm{keV}$ photopeak, a $6 \%$ upper scatter window set so its lower level abutted the upper level of the photopeak window and a $6 \%$ lower scatter window set so its upper level abutted the lower level of the photopeak window. For the psrf measurement, the triple-energy-window (TEW) scatter-estimation technique (Ogawa et al 1991) was used to obtain an estimate of high-energy-emission contamination of the photopeakwindow counts, that is, to obtain an estimate of the number of higher-energy (greater than $364 \mathrm{keV}$ ) photons that downscattered in the collimator septa, or that deposited less than their full energy in the camera crystal, and, as a result, were counted within the photopeak window. The TEW method can be used since both phenomena yield an energy spectrum that extends from above the photopeak window to below the photopeak window, as object scatter does. The estimate was subtracted from the total count in the photopeak window pixel by pixel. The resulting corrected psrf thus represented an estimate of 'good' (364 keV) I-131 counts. These counts are the only ones that are appropriately reconstructed using a patient/object attenuation correction corresponding to $364 \mathrm{keV}$ photons.

The phantom for the measurement of sphere activity as a function of sphere volume consisted of seven spheres located in a cylinder of elliptical cross section (Data Spectrum Corp., Chapel Hill, NC). The axis of the cylindrical phantom lay along the axis of rotation of the camera. The long axis of the elliptical cross section was $31.5 \mathrm{~cm}$, the short axis $23 \mathrm{~cm}$ and the height of the cylinder was $20.5 \mathrm{~cm}$. The spheres ranged in volume from 209 to $4.2 \mathrm{cc}$. They were arranged within the cylinder so as to be well separated. They were supported on posts that utilized the pre-drilled-and-tapped holes of the phantom. The largest sphere was located along the long axis of the elliptical cross section; its centre was $8 \mathrm{~cm}$ from one end cap of the cylinder. The other six spheres had their centres approximately in one plane that was $4.5 \mathrm{~cm}$ from the other end cap of the cylinder. Viewed from one end of the cylinder, the centres of six spheres were on the points of a hexagon and the seventh in the middle. The layout is shown in the reconstructed images of figure 3 . The activity concentration in the spheres was nearly the same, ranging between 70 and $79 \mathrm{kBq} \mathrm{cc}^{-1}$. The activity in the largest sphere at the beginning of the measurements was $15.5 \mathrm{MBq}$. The water solution in the cylinder had one of three levels of background activity that can be characterized by $b$, the ratio of the activity concentration in the water of the cylinder divided by that in the water of the largest sphere. The three values for $b$ were $0,0.21$ and 0.40 . The experiments were done in the order of increasing background, and took place over 5 days.

Seven point-source markers were distributed on the exterior, curvilinear surface of the cylinder containing the spheres during all SPECT acquisitions. Each consisted of a $3 \mathrm{~mm}$ diameter filter-paper disc saturated with a water solution of I-131. For each type of collimation, 
a fixed radius of rotation, a $120^{\circ}$ rotation, and six degrees between angular positions were employed for the SPECT acquisition. Sixty seconds were used for each projection angle. The data were acquired into a $64 \times 64$ projection array except for a separate test discussed below. Measurements were taken at a radius of rotation of 22 or $26 \mathrm{~cm}$. For these acquisitions, the data from the three windows were again analysed by the TEW method. Here the result was an estimate of the sum of object-scatter and high-energy-emission contamination of the photopeak-window counts.

Particular values of collimation type, radius of rotation and background level defined a given case. With UHE collimation, there were six cases in all: the three background levels at the $26 \mathrm{~cm}$ radius and the zero and $b=0.21$ background level at the $22 \mathrm{~cm}$ radius, with the last case repeated twice. With HE collimation, there were four cases in all: the three background levels at the $26 \mathrm{~cm}$ radius, and the zero background level at the $22 \mathrm{~cm}$ radius. Subsequent to the SPECT acquisitions, $1 \mathrm{~mm}$ diameter lead beads were placed on the top of and at the centre of the filter-paper discs, and a CT acquisition of the phantom obtained.

The total number of counts in the projection-image set was approximately 3.5 million with zero background, HE collimation and the $22 \mathrm{~cm}$ radius of rotation. About $84000(24 \%)$ of these were from the markers (this was $24 \%$ of all counts at zero background; it was a much smaller percentage at higher backgrounds as new activity was added to the cylinder while the marker and sphere activity both decayed). At a radius of $26 \mathrm{~cm}$, there were about $7.8 \%$ less total counts compared to the value at $22 \mathrm{~cm}$. For HE collimation and the $22 \mathrm{~cm}$ radius of rotation, there were approximately 16.7 million and 24.0 million total counts at the $b=0.21$ and 0.40 background levels, respectively. With zero background, UHE collimation and the $22 \mathrm{~cm}$ radius of rotation, there were approximately 1.2 million counts. This value is only $33 \%$ of the corresponding value with HE collimation because of the reduced sensitivity of the UHE collimation due to the thicker septa. Equivalent reductions occurred for the UHE collimation compared to the HE collimation for the other acquisition conditions.

For each particular case, after an initial filtered-backprojection reconstruction, we visually determined each marker's position in three dimensions in each modality. Then, the CT image (slice thickness $=3 \mathrm{~mm}$ ) was registered in three dimensions to the SPECT image using a program that minimized the sum of the distances between the paired marker locations. Next, a CT-space-to-SPECT-space transformation and an energy extrapolation of the CT values produced a $364 \mathrm{keV}$ attenuation map. This map was employed for attenuation correction in the subsequent 3D OSEM reconstruction. The 3D OSEM reconstruction also took account of the TEW estimate of photopeak-window contamination. The weights of the 3D transition matrix were calculated on the fly so RAM memory requirements were not a factor in carrying out a reconstruction. Reconstruction time on a Digital Equipment Corporation (Compaq) 500 au Personal Workstation was $1 \mathrm{~h} 10 \mathrm{~m}$ for 100 iterations of the UHE-collimated data and $2 \mathrm{~h} 5 \mathrm{~m}$ for the same number of iterations of the HE-collimated data that was reconstructed with a more complicated model. After reconstruction, the 3D OSEM image was retransformed into the CT space where geometric-sized volumes of interest (VoIs) were applied to yield total counts for each sphere.

The VoIs were determined in the CT space as follows. The central plane of the largest sphere was determined visually. Using that central plane, the centre and radius of the circle that visually matched the interior edge of the sphere best were determined. This circle contained 2292 pixels (the original $512 \times 512 \mathrm{CT}$ had been resampled at $256 \times 256$ ). The centre and radius were then employed within an in-house computer program to determine a spherical VoI that could be used in a three-dimensional image. The total number of pixels for this VoI was noted. The central plane for the other spheres was again determined visually. The number of pixels each circle should have was calculated from the geometrical volume of each sphere, 
relative to the geometrical volume of the largest sphere, and the noted total number of pixels in the circle of the largest sphere. Given the size of each circle, its location in a sphere's central plane was chosen visually. VoIs were then created for each sphere using the in-house computer program.

The VoIs were used for a final adjustment to the registration. In the majority of cases, this final adjustment was separately determined for each VoI, based on obtaining the maximum total counts in the VoI ( $\mathrm{Li}$ and Koral 2001). In one case, it was based on all six VoIs (the smallest sphere was ignored-see below). After each adjustment, the corresponding sphere VoI was applied to the resultant SPECT-space-to-CT-space transformation of the 3D OSEM reconstruction to yield the total count for the sphere. In a test with the case employing a registration adjustment based on all six VoIs, using individual adjustment increased the total count by not more than $2 \%$ for any sphere, compared to the slightly-more-realistic adjustment based on all six.

The activity recovery coefficient as a function of sphere volume was defined as

$$
\mathrm{RC}(V)=\frac{A_{\mathrm{m}}(V)}{A_{\mathrm{t}}(V)}
$$

where $A_{\mathrm{m}}(V)$ is the estimated activity from the measurement of the sphere with volume $V$ and $A_{\mathrm{t}}(V)$ is the true activity for that sphere. The value for $A_{\mathrm{m}}(V)$ came from the measurement of total counts reconstructed within a spherical VoI by dividing the measured value by a countsto-activity conversion factor, $\mathrm{CF}$. This $\mathrm{CF}$ is sometimes called the camera calibration factor. In the present measurements, for each case, the reconstructed counts in the largest sphere and its true activity were used to determine the CF value. That is,

$$
\mathrm{CF}=\frac{C_{\mathrm{m}}(209 \mathrm{cc})}{A_{\mathrm{t}}(209 \mathrm{cc})}
$$

where $C_{\mathrm{m}}(209 \mathrm{cc})$ is the measured total count in the $209 \mathrm{cc}$ sphere and $A_{\mathrm{t}}(209 \mathrm{cc})$ is its true activity at the time of the measurement for the particular case. (The true activity of each sphere is determined by decay correcting the value for the activity injected into it. This activity was determined by a dose-calibrator measurement at a given time with a subtraction for the amount of activity left in the syringe.) Our procedure yields $100 \%$ recovery for the largest sphere for each collimation-radius-background case.

\section{Test with finer sampling and smaller voxel}

With the $b=0.21$ background and using HE collimation, we repeated the acquisition using $128 \times 128$ projection sampling. Other acquisition parameters were unchanged. The same HE model as that used for $64 \times 64$ sampling was set up for the finer sampling, and an image voxel $3.8 \mathrm{~mm}$ on a side used, instead of one $7.6 \mathrm{~mm}$ on a side. Reconstruction with 3D OSEM was then carried out as usual.

\section{Application of RC-based correction to activities in spherical tumours in a patient simulation}

Previously, a separate study had simulated projection data for a 'voxel man' containing a realistic I-131 activity distribution and spherical 'tumours' of various volumes located in the abdomen (Dewaraja et al 2005). The voxel-man phantom was a $128 \times 128 \times 60$ matrix with a $4 \mathrm{~mm}$ voxel. The study employed both HE and UHE collimation and the Prism camera. The true activity values were based on radioimmunotherapy-patient data from imaging on the second day of therapy. The relative true activity concentrations were: tumours, 25; blood, 12; liver, 7; lung, 7; spleen, 13, and rest of body, 1. The four spherical tumours were placed 
randomly within the abdomen but not within or touching an organ. Their volumes were 135, 59, 16 and $7 \mathrm{cc}$. Total activity in the camera field-of-view was set at $1 \mathrm{GBq}$. A low-noise projection image set for the voxel man with four tumours was simulated by Monte Carlo. The simulation included the effects of attenuation and scatter within the voxel man who has a non-uniform distribution of attenuation coefficients. The simulations employed $360^{\circ}, 60$ angles and triple energy windows as in the present study. The projection-image matrix was $128 \times 128$ (a $4 \mathrm{~mm} \times 4 \mathrm{~mm}$ pixel). Twenty realizations of the phantom were generated by adding Gaussian noise to the original low-noise projection set. The 20 projection sets were reconstructed with 3D OSEM using a psrf model generated by fits to a simulated psrf for a set of distances of the point source from the detector. The models employed used the same functions as in this study. Six subsets and 60 iterations were employed. Attenuation correction was included in the reconstruction, as well as compensation for scatter. The scatter estimate was generated using the triple-energy-window method as in this study. Total counts within each tumour were obtained by summation over geometric-sized VoIs.

A separate simulation of a large sphere centred within an elliptical-cross-section cylindrical phantom containing background was carried out. It was similarly reconstructed and the $\mathrm{CF}$ value calculated from the total counts within the sphere's geometrical VoI and its known simulated activity. Using this CF value, SPECT-estimated activities were obtained from the total count for each of the tumours in each reconstruction. The resultant values were averaged over the 20 realizations. The mean estimated-activity values were compared to the true activities in the previous publication. In this paper, that comparison is repeated in table 3 and an $\mathrm{RC}$ value is calculated for each tumour volume from the fit to the average RC versus $V$ curve from the new phantom measurements. The previously-estimated activity is then divided by this $\mathrm{RC}$ value to correct the activity estimate. Then these corrected activity estimates are compared to the true activities in the same table.

\section{Results}

\section{Psrf}

With the high rate of sampling of the projection image, the corrected I-131 psrf with either collimation exhibited a central pattern of hot spots corresponding to the hexagonal arrangement of the collimator holes. The peak of the hot spots had diminishing amplitude as one moved out from the central peak, that most nearly opposite the source. This central pattern changed as the distance of the point source changed, with more 'hexagonal rings' added as the distance of the point source from the camera increased. A magnified image of the central pattern for the HE collimator with the point source $13 \mathrm{~cm}$ in front of the camera is shown in figure 1(a). All count levels at 59 to $100 \%$ of the maximum count are set to the brightest intensity so that lower intensities are better visualized. The hexagonal arrangement of the peaks is apparent. A similar image for the UHE collimator with the point source $24.5 \mathrm{~cm}$ in front of the camera was published previously (Koral et al 2004).

With the HE collimator, the psrf image also had a six-ray septal-penetration star pattern of significant intensity. The septal-penetration star pattern with the UHE collimator was much less intense because of its much thicker septa. The star pattern for the HE collimator is shown in figure 1(b), which is the same image as in figure 1(a) but without magnification and with the grey scale changed to show even smaller count values. Now, the spots of the central pattern are blurred together, and the lower-count ray structure, with its hexagonal symmetry, is visualized. The edge of the entire pattern is defined by the rectangular shape of the camera crystal. 


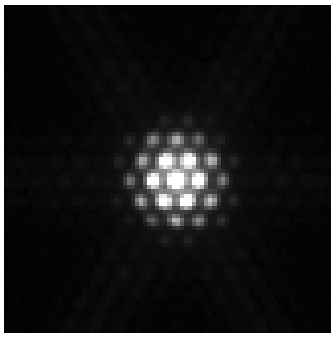

(a)

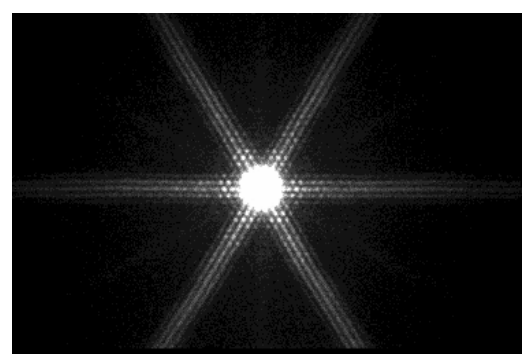

(b)

Figure 1. (a) Magnified $(2.3 \times)$ central portion of the HE-collimator psrf for a source $13 \mathrm{~cm}$ in front of the camera. (b) Same HE-collimator psrf as in (a) but unmagnified and with the low intensities made visible.

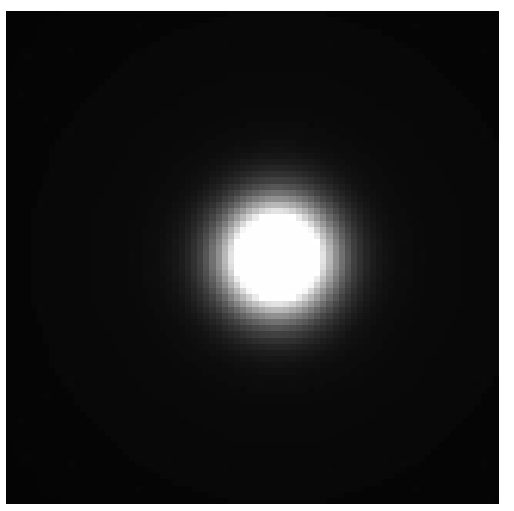

Figure 2. Magnified central portion of the model psrf at $13 \mathrm{~cm}$.

With $64 \times 64$ or $128 \times 128$ sampling, the fine structure of the central pattern is much less clear. For this reason, and to keep the model of the collimator-detector response simple, we used approximations to the psrf patterns. That is, we employed a simple, smooth function that matched averages of the count distribution. For the HE collimation, a rotationally-symmetric Gaussian that was centred on the peak most nearly opposite the source accounted for the main part of the response, and a rotationally-symmetric two-sided decaying exponential accounted for the circularly-averaged level of the septal-penetration star pattern as a function of the radial distance from the central peak. For each distance, parameters for the model were obtained by a two-dimensional least-squares fit to the data. An image of the approximation for the $13 \mathrm{~cm}$ distance is shown in figure 2. The brightest intensity is set in the same way as it was in figure 1(a). The smoothness and rotational symmetry of the approximation is apparent. For the UHE collimation, we neglected the very-low-intensity star pattern and employed only a Gaussian for the model. The number of total counts lost was less than $1.2 \%$ and so the effect of their neglect should be minimal. A similar description of the fitting process including a plot of the fit to the data points has been given previously (Koral et al 2001, 2004, 2005). For each collimation type, the 3D model for the OSEM reconstruction transition matrix was determined by interpolating the fits to the psrf at the given depths to intermediate distances. 

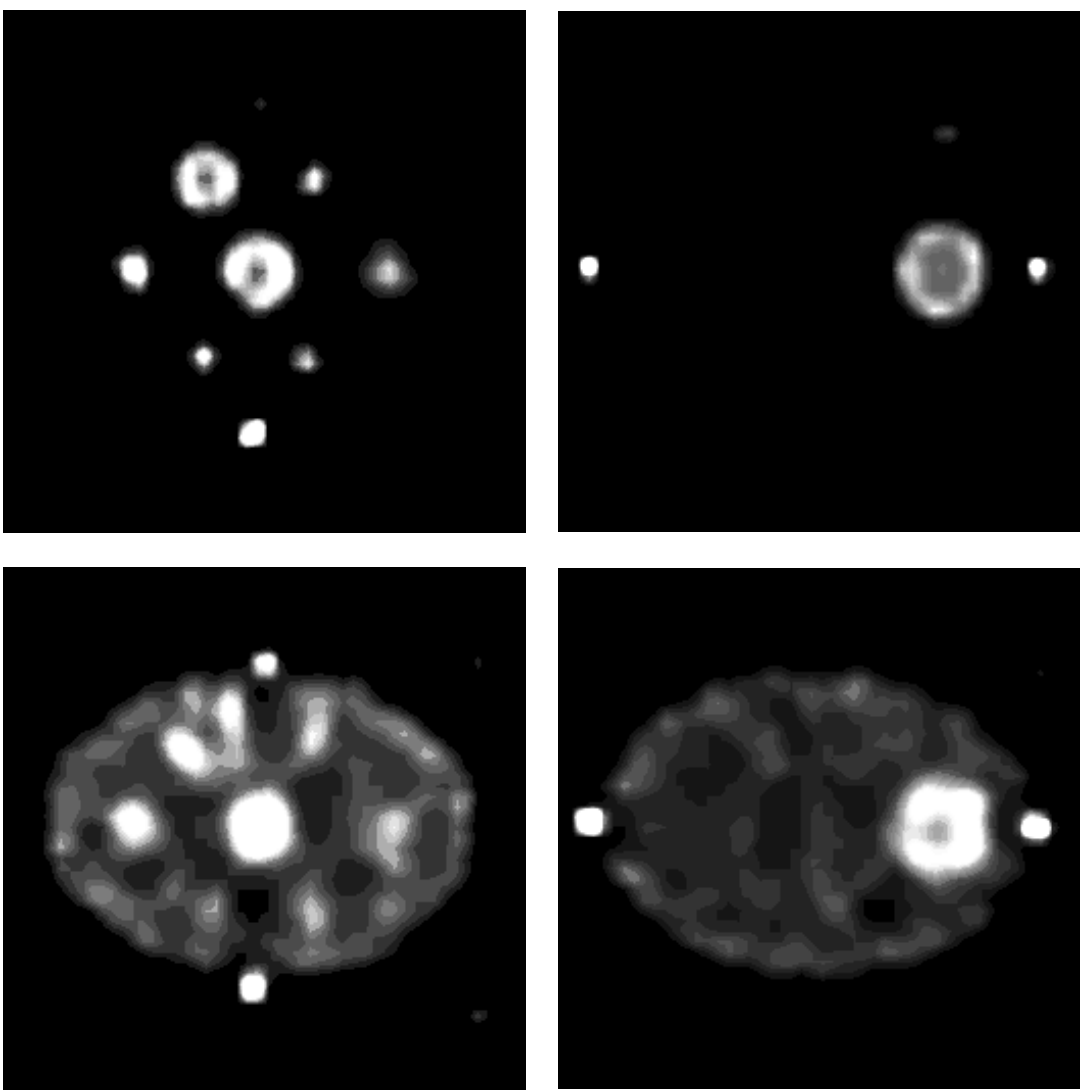

Figure 3. Reconstructed images after 100 iterations with six subsets. At top, background is zero, while at bottom it is non-zero. At left, the transverse plane passes approximately through the centres of the six smaller spheres. At right, the plane passes approximately through the centre of the largest sphere.

SPECT imaging and conversion factor. The projection data were divided into six subsets for all reconstructions of the phantom data. Resultant images of the phantom were investigated qualitatively and quantitatively out to 100 iterations. The appearance of the images became noisier as the iteration number increased, but the counts in each sphere showed a regular increase. The way the image becomes noisier as the iteration number increases with a similar model has been shown for different data in a related publication (Koral et al 2006). Two 100-iteration reconstructions of the present HE data, one with $b=0$ (top) and the other with $b=0.21$ (bottom), are shown in figure 3. At the left is the plane that approximately passes through the centre of the six smaller spheres (only an edge of the largest sphere appears, located at 3 o'clock). The $90.9 \mathrm{cc}$ sphere is at the centre. The next larger sphere with a volume of $62.8 \mathrm{cc}$ is at 11 o'clock and the $16.4 \mathrm{cc}$ sphere is at 9 o'clock. The three smallest spheres appear at 1, 5 and 7 o'clock. At the right is the plane through the centre of the largest sphere. The hot spots at the phantom edges are markers.

Figure 3 shows that the largest sphere clearly exhibited an edge overshoot (alternately described as a count-deficient centre) with or without cylinder background activity. For zero background, which represents a simpler object than that with non-zero background, there appears to have been a sufficient number of iterations for the overshoot to occur for the next 
Table 1. Difference in recovery coefficient averaged over all non-zero-background cases and that averaged over all zero-background cases, as a function of sphere volume. Presented for both HE and UHE collimation.

\begin{tabular}{|c|c|c|c|c|c|c|}
\hline \multirow{3}{*}{ Collimation } & \multicolumn{6}{|c|}{$\begin{array}{c}\text { Difference }(V) \text { in } \mathrm{RC}(V) \text { between non-zero- } \\
\text { and zero-background cases }\end{array}$} \\
\hline & \multicolumn{6}{|c|}{ Volume $V(\mathrm{cc})$} \\
\hline & 209 & 90.9 & 62.8 & 16.4 & 11.2 & 8.1 \\
\hline $\mathrm{HE}$ & 0.00 & 0.090 & 0.189 & 0.019 & 0.087 & 0.062 \\
\hline UHE & 0.00 & 0.014 & 0.170 & 0.091 & 0.231 & 0.058 \\
\hline
\end{tabular}

two smaller sphere sizes as well. For non-zero background, only the smaller of the two has an overshoot, and it is not circumferentially symmetric. The general way the overshoot for a particular-size sphere first shows up and then changes as the iteration number increases has been shown with a similar model for different data in the related publication cited earlier (Koral et al 2006). Unpublished simulation results indicate that for a given background the edge overshoot changes with the size of the object containing the uniformly-distributed activity compared to the full width at half maximum of the system psrf; at a given number of iterations, it can be non-existent for particular values of that ratio. The occurrence of an overshoot appears to be a characteristic of maximum-likelihood reconstruction. It obviously has an impact on the accuracy of the estimate of the activity distribution. Further investigation of the phenomenon is ongoing.

In spite of noise in the image, we determined that the total count in each sphere had successfully attained an asymptotic value by 100 iterations. This was true for all sphere sizes except the smallest sphere, for which the value for total counts was still increasing. We, therefore, used 100 iterations for all our phantom results herein. However, we did not determine an estimate of activity recovery for the smallest sphere since the lack of convergence would bias the activity-recovery value.

For what follows, we calculated individual CF values for each case. We have previously shown that with 3D OSEM reconstruction the CF value decreases only slightly as the $b$ value increases. Typically, the CF decreases by only $6.0 \%$ over the $b$ range of 0 to 0.4 for UHE collimation (Koral et al 2004) and by $4.2 \%$ for HE collimation (Koral et al 2005). Since individual CFs' values are not much different, an average CF would have yielded only slightly different results compared to those presented here. Incidentally, it has also been shown that reconstructing a (1) known-activity $200 \mathrm{cc}$ sphere in a large cylinder, (2) a large cylinder with a known activity or (3) a point source and using a VoI radius of between 2 and 4 voxels all yield a similar value for the counts-to-activity conversion factor for a given camera-collimatorwindow-setting combination (Dewaraja et al 2005).

\section{Dependence of $R C$ on radius of rotation, background level}

We were not able to discern any consistent dependence of the recovery coefficient on the radius of rotation of the circular orbit. There was a considerable difference between results with zero background, and those at the other two levels of background. For each collimator, we averaged the zero background cases, and compared them to the average of the non-zerobackground cases. The results are given in table 1. For HE collimation, the average difference in recovery coefficient over the five smaller volumes is 0.089 , and for UHE collimation, 0.113 . The differences are consistent with the known dependence of the results from 3D OSEM reconstruction on the complexity of the object; they can be characterized as a tendency for 
Table 2. Recovery coefficient averaged over all cases using HE collimation and that averaged over all cases using UHE collimation, and the difference between the average values, as a function of sphere volume.

\begin{tabular}{llllllr}
\hline & \multicolumn{5}{c}{ Volume $V(\mathrm{cc})$} \\
\cline { 2 - 7 } Parameter & 209 & 90.9 & 62.8 & 16.4 & 11.2 & 8.1 \\
\hline UHE RC & 1.00 & $0.952( \pm 0.022)$ & $0.848( \pm 0.089)$ & $0.883( \pm 0.060)$ & $0.603( \pm 0.122)$ & $0.779( \pm 0.133)$ \\
HE RC & 1.00 & $0.925( \pm 0.052)$ & $0.895( \pm 0.109)$ & $0.857( \pm 0.039)$ & $0.700( \pm 0.051)$ & $0.790( \pm 0.041)$ \\
Difference $(V)$ & 0 & 0.027 & -0.047 & 0.025 & -0.097 & -0.011 \\
\hline
\end{tabular}

Difference $(V)=\overline{\mathrm{R}} \overline{\mathrm{C}}_{\mathrm{UHE}}(V)-\overline{\mathrm{R}} \overline{\mathrm{C}}_{\mathrm{HE}}(V)$.

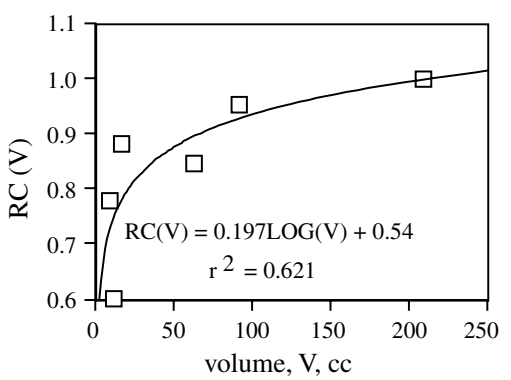

Figure 4. Recovery coefficient, averaged over all cases measured with UHE collimation, versus volume.

activity to be recovered less well in focal regions when there are a greater number of voxels in which counts can reasonably be placed.

\section{Dependence of $R C$ on type of collimation}

We also averaged $\mathrm{RC}(V)$ values over all cases with each type of collimation. The mean (and standard deviation in parentheses) are given for each volume in table 2 . The average values were compared by taking their difference. The results are given in the bottom row of table 2 . The $\mathrm{RC}(V)$ values are quite similar at each volume, with the difference usually being less than at least one of the two standard deviations, and sometimes less than both. The average of the difference over the five volumes with non-zero results is only -0.020 .

A plot of $\overline{\mathrm{R}} \overline{\mathrm{C}}_{\mathrm{UHE}}(V)$ versus $V$ for UHE collimation is presented in figure 4 . The fitted function in the figure will serve as a calibration curve for RC-based correction in the patient simulations using UHE collimation.

A similar plot and curve were produced for HE collimation. They are shown at the top of figure 5. The fitted function will serve as the calibration curve in the patient simulations using HE collimation.

Comparison of recovery coefficients from images reconstructed by 3D OSEM to similar results from images reconstructed by $1 D S A G E$

Results for the same scintillation camera with the same HE collimation were published previously for 1D SAGE reconstruction (Koral and Dewaraja 1999). In that research, we used six spheres that ranged in volume from 200 to $1.6 \mathrm{~cm}^{3}$ placed in the same cylindrical phantom. For the RC evaluation, the counts-to-activity conversion factor was determined in a separate measurement and its dependence on the background level was determined. As a 


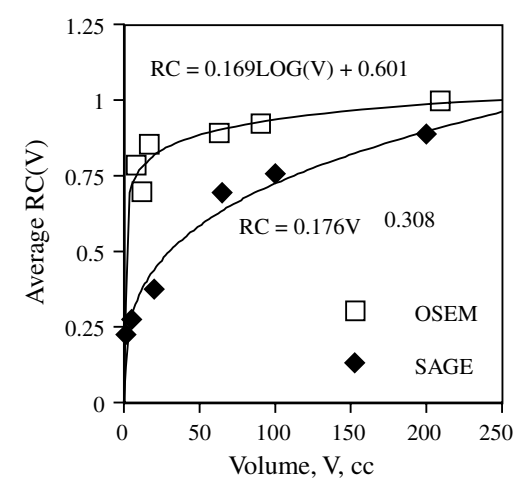

Figure 5. Comparison of activity recovery with HE collimation employing 3D OSEM or 1D SAGE.

consequence, the RC for the largest sphere was not necessarily $100 \%$ for a background-levelradius combination. In addition, no dependence on background level was observed but one was seen for radius. The recovery coefficients we plot in figure 5 for comparison to our new results with 3D OSEM are those with the radius equal to $27.5 \mathrm{~cm}$. The $\mathrm{RC}(V)$ values at this radius from $1 \mathrm{D}$ SAGE were $0.89,0.76,0.70,0.38,0.27$ and 0.22 for the $200,100,65.1,20,5.5$ and $1.6 \mathrm{cc}$ spheres, respectively. The plot is the bottom one in figure 5. The coefficients were only slightly higher for the other radius. The increase ranged from 0.11 for the $200 \mathrm{cc}$ sphere down to 0.055 for the $1.63 \mathrm{cc}$ sphere (Koral and Dewaraja 1999). For our new measurements with 3D OSEM reconstruction, we could not discern differences in activity recovery between the two radii of rotation as mentioned previously. Nor could differences be discerned between the two non-zero background levels $(b=0.2$ and $b=0.4)$. Therefore, it was appropriate to use the average of the RC values from all four cases for the comparison to the results from 1D SAGE. Doing so, we obtained the RC values shown in figure 5. In contrast to the 1D SAGE experiment, the counts-to-activity conversion factor was determined from the average values for the largest sphere, and so the RC at $208 \mathrm{cc}$ is exactly 1.00 . Figure 5 shows that a large improvement is achieved in recovering total activity by using 3D OSEM rather than 1D SAGE.

\section{Effect of applying RC-based correction to activities of spherical tumours in a patient} simulation

Since the background in the patient simulation was not uniform, it did not match any of the backgrounds in the $\mathrm{RC}(V)$ versus $V$ measurements. Therefore, we used the curves with the $\mathrm{RC}(V)$ values averaged over all cases discussed earlier. We evaluated the correction $\mathrm{RC}$ from the fit equation at the volume of each simulated tumour. Then, we corrected the estimated activity for each tumour by dividing by the corresponding RC value. The results of the correction for the two types of collimation are shown in table 3 . They are compared to the previous results with no correction. The activity estimates are moderately improved for both types of collimation.

\section{Effect of finer sampling and smaller voxel}

The $128 \times 128$ projection sampling combined with the $3.8 \mathrm{~mm}$ image voxel increased the measured recovery coefficient for three of four volumes for which we had comparison 


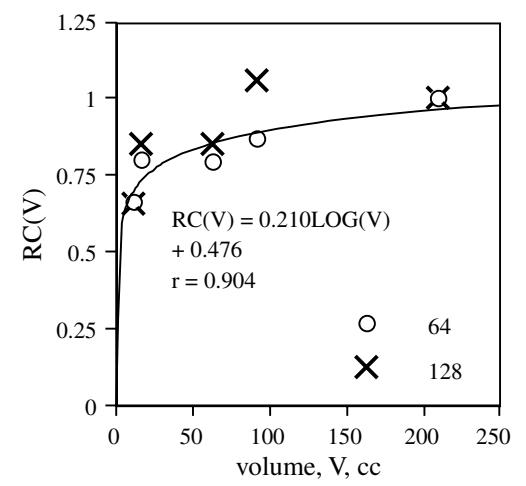

Figure 6. Effect of sampling rate on activity recovery with 3D OSEM.

Table 3. Individual errors in SPECT estimate of tumour activity before and post RC-based correction and average of absolute value of those errors. Presented for both HE and UHE collimation.

\begin{tabular}{|c|c|c|c|c|c|}
\hline \multirow[b]{2}{*}{$\begin{array}{l}\text { Tumour } \\
\text { volume (cc) }\end{array}$} & \multirow[b]{2}{*}{$\begin{array}{l}\text { True activity } \\
\text { (MBq) }\end{array}$} & \multicolumn{2}{|c|}{ SPECT-estimated } & \multicolumn{2}{|c|}{ Post-correction } \\
\hline & & $\begin{array}{l}\text { Activity } \\
(\mathrm{MBq})\end{array}$ & Error \% & $\begin{array}{l}\text { Activity } \\
(\mathrm{MBq})\end{array}$ & Error \% \\
\hline \multicolumn{6}{|c|}{ HE collimation } \\
\hline 135 & 52.8 & 52.9 & 0.2 & 55.1 & 4.3 \\
\hline 59 & 23.0 & 21.4 & -6.8 & 23.5 & 3.5 \\
\hline 16 & 6.40 & 5.7 & -10.5 & 7.12 & 11.2 \\
\hline 7 & 2.90 & 1.6 & -43.3 & 2.21 & -23.8 \\
\hline Average & & & 15.2 & & 10.7 \\
\hline \multicolumn{6}{|c|}{ UHE collimation } \\
\hline 135 & 52.8 & 53.0 & 0.5 & 55.3 & 4.7 \\
\hline 59 & 23.0 & 20.6 & -10.3 & 23.2 & 0.9 \\
\hline 16 & 6.40 & 5.9 & -7.9 & 7.58 & 18.5 \\
\hline 7 & 2.90 & 1.8 & -38.4 & 2.53 & -12.8 \\
\hline Average & & & 14.3 & & 9.2 \\
\hline
\end{tabular}

Error $=100 \% *\{($ estimated - true $) /$ true $\}$.

reconstructions. It was unchanged for the $209 \mathrm{cc}$ sphere because of the way the activity calibration was carried out. The results for RC versus $V$ are shown in figure 6 . The difference ranged from a fairly large increase of 0.185 for the $90.9 \mathrm{cc}$ sphere to a slight decrease of 0.0070 for the $11.2 \mathrm{cc}$ sphere, the smallest volume in the plot. Averaged over the four sphere volumes the difference equalled 0.071 .

\section{Discussion}

In the HE collimation case, the activity recovery was considerably better using 3D OSEM reconstruction than it had been in the past using 1D SAGE reconstruction (figure 5). This result implies that modelling the collimator-detector response in 3D improves the resolution of I-131 imaging as had been indicated by previous large-sphere measurements, and had been shown previously for Tc-99m imaging. The difference in the reconstruction algorithm probably played a role as well, since SAGE is a regularized algorithm while the basic form 
of OSEM that we used is not regularized, and no post-reconstruction filtering was employed. We think the role of the reconstruction algorithm is minor compared to the role played by the $3 \mathrm{D}$ modelling of the psrf, but that has not been proven.

A general concern with using correction based on RC values is that the correction is determined with one geometry and one set of acquisition parameters, but is applied to another geometry and perhaps a different set of acquisition parameters. A method to obviate the concern is to use a newly-introduced 'partial-volume' correction (PVC) that is particular to each geometry of interest. Perturbation-based forms of PVC were introduced for brain imaging with In-111 (Du et al 2005). Good activity quantification was obtained with such partial-volume compensation in simulations of Tc-99m imaging employing reconstruction that did not include 3D modelling of the psrf (Boening et al 2004). Some of the compensated Tc-99m images exhibited a dark ring about the focal volume, but that ring did not appear to interfere with activity quantification within the target (Boening et al 2004). Perturbationbased partial-volume compensation has also yielded good quantitative results for high-count I-131 imaging employing 3D OSEM reconstruction (Dewaraja et al 2006). 'Visual image quality also improved significantly after the correction' (Dewaraja et al 2006). However, in the research cited the uniform activity distributions in the targets and in the background matched the assumption of uniform distributions in the feature templates of PVC. Therefore, it is not yet clear how well PVC will perform if the activity is distributed non-uniformly, or if the imaging is low count. After I-131 partial-volume correction becomes better established, a head-to-head comparison of results from it to those from a correction based on recovery coefficients will be in order.

The increase in activity recovered with finer projection sampling and a smaller voxel suggests that a future measurement of a calibration plot of recovery coefficient versus volume with finer projection sampling and a smaller voxel might yield a larger improvement in the corrected activities than that which has been demonstrated in this paper. On the other hand, applying an RC-based correction that is based on spheres to uniform-activity tumours that are non-spherical in future patient simulations might result in decreased accuracy. Non-uniform activity distributions in tumours of all shapes also need to be investigated in the future.

\section{Conclusions}

When the 3D model used in reconstruction is adjusted for the type of collimation, recovery of the I-131 activity in spheres of various sizes placed within a cylindrical phantom and imaged with ultra-high-energy collimation is similar to that achieved with high-energy collimation. Activity corrections based on phantom-based recovery coefficients successfully reduce the bias of tumour-activity estimates in simulations of patient SPECT imaging. In the case of I-131, it remains to be determined whether such recovery-coefficient-based, or partial-volume corrections are more effective for the range of cases observed in the clinic.

\section{References}

Beekman F J, Slijpen E T and Niessen W J 1998 Selection of task-dependent diffusion filters for the post-processing of SPECT images Phys. Med. Biol. 43 1713-30

Boening G, Pretorius P H and King M A 2004 Study of relative quantification of Tc-99m with partial volume effect and spillover correction for SPECT oncology imaging IEEE Nucl. Sci. Symp. Med. Imaging Conf. (Rome, Italy, 2004) CD ROM IEEE 2004

Dewaraja Y K, Ljungberg M and Fessler J A 2006 Anatomical information based partial volume compensation for I-131 SPECT imaging in radioimmunotherapy $J$. Nucl. Med. 47 115P-6P (supplement 1, abstract book) 
Dewaraja Y K, Wilderman S J, Ljungberg M and Koral K F 2005 Accurate dosimetry in I-131 radionuclide therapy using patient specific, 3-dimensional methods for SPECT reconstruction and absorbed dose calculation J. Nucl. Med. 46 840-9

Du Y, Tsui B M W and Frey E C 2005 Partial volume effect compensation for quantitative brain SPECT imaging IEEE Trans. Med. Imaging 24 969-76

Fessler J A and Hero A O 1994 Space-alternating generalized expectation-maximization algorithm IEEE Trans. Signal Process. 42 2664-77

Hudson H M and Larkin R S 1994 Accelerated image-reconstruction using ordered subsets of projection data IEEE Trans. Med. Imaging 13 601-9

Koral K F and Dewaraja Y 1999 I-131 SPECT activity recovery coefficients with implicit or triple-energy-window scatter correction Nucl. Instrum. Methods Phys. Res. A 422 688-92

Koral K F, Dewaraja Y, Li J, Lin Q, Regan D D, Zasadny K R, Rommelfanger S G, Francis I R, Kaminski M S and Wahl R L 2003 Update on hybrid conjugate-view SPECT tumor dosimetry and response in 131I-tositumomab therapy of previously untreated lymphoma patients J. Nucl. Med. 44 457-64

Koral K F, Kritzman J N, Rogers V E, Ackermann R J and Fessler J A 2006 Optimizing the number of equivalent iterations of 3D OSEM in SPECT reconstruction of I-131 focal activities Nucl. Instrum. Methods Phys. Res. A at press

Koral K F, Lin Q, Yendiki A, Akhtar A, Dewaraja Y and Fessler J A 2001 Effect of 3-D detector response on SPECT quantification of focal I-131 activity IEEE Nucl. Sci. Symp. Med. Imaging Conf. (San Diego, CA, 2001) (CD ROM published in New York: IEEE, 2001)

Koral K F, Yendiki A, Lin Q and Dewaraja Y K 2005 Comparison of 3D OSEM versus 1D SAGE for focal total-activity quantification in I-131 SPECT with HE collimation IEEE Trans. Nucl. Sci. 52 154-8

Koral K F, Yendiki A, Lin Q, Dewaraja Y K and Fessler J A 2004 Determining total I-131 activity within a VoI using SPECT, a UHE collimator, OSEM, and a constant conversion factor IEEE Trans. Nucl. Sci. 51 611-8

Li J and Koral K F 2001 An algorithm to adjust a rigid CT-to-SPECT fusion so as to maximize tumor counts from CT VoI in 131-I therapies IEEE Nucl. Sci. Symp. Med. Imaging Conf. (San Diego, CA, 2001) (CD ROM published in New York: IEEE, 2001)

Ogawa K, Harata H, Ichihara T, Kubo A and Hashimoto S 1991 A practical method for position dependent Comptonscatter correction in single photon emission CT IEEE Trans. Med. Imaging 10 408-12

Vija A and Engdahl J 2003 Analysis of a SPECT OSEM reconstruction method with 3D beam modeling and optional attenuation correction: phantom studies IEEE Nucl. Sci. Symp. Med. Imaging Conf. (Portland, OR, 2003)

Zeng G L and Gullberg G T 1992 Frequency-domain implementation of the 3-dimensional geometric point response correction in spect imaging IEEE Trans. Nucl. Sci. 39 1444-53

Zeng G L, Gullberg G T and Tsui B M W 1991 Three-dimensional iterative reconstructive algorithms with attenuation and geometric point response correction IEEE Trans. Nucl. Sci. 38 693-702 